\title{
How Should You Frame Questions to Measure User Attitudes Accurately? An Experimental Design Study
}

\author{
Seema Swamy \\ 10828 Alderbrook Lane, Cupertino, CA-95014 \\ seemavasanth@yahoo.com
}

\begin{abstract}
Attitudes are most frequently measured through responses to questionnaires. The validity of the results is strongly dependent on the quality of the instrument. Questionnaires employed to evaluate users' attitudes toward issues, products, or services frequently tend to have several biases yielding inflated responses. A 2 × 2 experimental design study established that rating attitudinal items on a Likert scale in a questionnaire with all statements framed in positive valence tend to produce higher attitudinal scores than when statements are balanced with both positive and negative valence.
\end{abstract}

Keywords: Attitude measurement, validity, reliability, survey, experimental design, questionnaire, and Likert scale.

\section{Introduction}

Attitude measurement is the basis of consumer and market research and is central to the field of social sciences [3, 8]. As Gordon Allport stated in the Handbook of Social Psychology, "An attitude is a mental and neural state of readiness, organized through experience, exerting a directive or dynamic influence upon the individual's response to all objects and situations with which it is related [1]."

Attitudes clearly exist, but can rarely be observed and measured directly. They are often considered precursors of conscious behaviors. The measurement of attitudes within individuals is extremely important as they determine product loyalty and brand perceptions, affecting actions such as purchasing products or services, and the quality of future interactions.

Users' perceptions and attitudes are most often measured through self-reports obtained through questionnaires. Questionnaires tend to have closed-ended items in which respondents are instructed to select their responses based on a list of options provided [4]. Closed-ended questionnaires are generally cheaper, more reliable, and faster to code and analyze the data collected.

\section{Literature Review}

Questionnaires should be designed not only to ensure that the constructs measured are reliable, but also to maximize the accuracy of the measured attitudes. Frequently, in market research and consumer service surveys, participants are asked to state their 
agreement to a set of statements on a number of issues. One of the most common biases evident in these questionnaires is that the statements are frequently framed with only positive affect without balancing it with statements framed in negative affect, thereby introducing an acquiescence bias.

Meaningless acquiescence to attitude items by the sample of respondents is not generalizable to the population [7]. Concerns over the acquiescent response set bias first became prominent in the measurement of attitudes in the California $\mathrm{F}$ scale research [5]. In addition to acquiescence bias, respondents are also less likely to read the statements carefully when all the statements are framed positively. When the questionnaire is balanced with both positive and negative valence items, participants are compelled to read each of the statements more carefully to rate them consistently.

Most research that has tested best practices in research methods has employed college students as participants. This has frequently been cited as a limitation in the generalization of findings about optimal questionnaire construction to other populations.

Therefore, for this study, the target sample consisted of users of a customer-tocustomer e-commerce site where sellers and buyers interact with each other to transact products and services. When the users of this e-commerce site encounter any problem or issue, they contact customer support. The most frequent form of communication is through email. To assess the existence of acquiescence bias, attitudes in three main areas were measured - the artifact itself, that is, the email response provided by the customer support representative (CSR), the responder of the email, that is, the customer support representative, and how valued the recipient of the email felt based on the reply of the CSR.

Hypothesis 1: When a questionnaire is designed with statements framed in the affirmative or with only positive valence, the responses will be significantly more positive than when the statements are balanced with both positive and negative valence.

Sub-hypothesis la: The positive bias of affirmative questionnaire would lead to more positive evaluation of artifacts such as emails compared to evaluations obtained through balanced questionnaire.

Sub-hypothesis 1b: The positive bias of affirmative questionnaire would lead to more positive evaluation of the responder of the emailed question compared to evaluation obtained through balanced questionnaire.

Sub-hypothesis 1c: Evaluation of how valued the recipient of the email response felt would be significantly more positive when it is measured with the affirmative questionnaire than when it is measured with a balanced questionnaire.

Two types of emails were employed to ensure that the effects of questionnaire design were not due to the type of email issue and response. The two types of emails were - one pertained to a policy of the customer-to-customer e-commerce site and the second pertained to the transgression of a partner transacting on the site. Generally, individuals tend to have stronger affect toward another individual due to social presence [6] compared to a more diffuse entity such as a company policy of relatively 
less consequence. Thus, negative experiences with another transacting user would likely engender a relatively more negative perception compared to an issue with a company policy.

Hypothesis 2a: Individuals will rate an email more positively when the issue pertains to a clarification of a company policy than when the issue pertains to a transgression by a transacting partner.

Hypothesis 2b: Individuals will rate the responder of an email more positively when the issue pertains to a clarification of a company policy than when the issue pertains to a transgression by a transacting partner.

Hypothesis 2c: Individuals will rate how valued they felt significantly more when the issue pertains to a clarification of a company policy than when the issue pertains to a transgression by a transacting partner.

\section{Method}

\subsection{Participants}

1133 participants participated in the between-subjects experimental design study. Participants were users of a customer-to-customer e-commerce site.

\subsection{Procedure}

In the $2 \times 2$ between-subjects experimental design study (type of questionnaire vs. type of email issue), participants were asked to assume that they had written a question to the CSR and had received a response. The study was run online. Participants were provided with an online copy of a question written to customer support and a response to the question written by a customer support representative.

Following this, they were asked to complete a questionnaire online. The questionnaire consisted of statements about perceptions of the email, perceptions of the CSR, and how valued the user of the customer-to-customer e-commerce site felt after reading the response. Participants were asked to indicate the extent to which they agreed or disagreed with the statements.

There were two types of questionnaires - one with affirmative statements and other with balanced statements. In the affirmative questionnaire, participants received a questionnaire with all items constructed in positive valence. In the balanced questionnaire, the items contained both positive and negative valence statements. Participants were randomly assigned to provide their evaluations on one type of questionnaire.

There were two types of emails employed in the study - one related to the billing policy and the second related to the behavior of a transacting partner on the customerto-customer e-commerce site. The billing policy issue pertained to a general support question about fees charged by the e-commerce site. A customer-to-customer e-commerce site seller wrote an email inquiring about a \$1.65 charge to his account and was mistaken that this amount was a monthly charge. The CSR clarified that the charges were related to two items he had listed on the site and that it was not a recurring charge as there was no monthly fee. The second email pertained to a seller's 
negative experience with a buyer on the site. Specifically, the issue pertained to a complaint from a seller about a bidder who backed out of a winning bid. The CSR responded that appropriate action was taken on the transgressing partner and offered credit to the complainant for listing the item on the site. Participants were randomly assigned to receive one type of emailed question and response.

The online questionnaire consisted of attitudinal measures. Participants were asked to rate their degree of agreement to a set of statements on a 5-point Likert scale ranging from Strongly Disagree to Strongly Agree. The statements appeared in a random order to control for order effects [2]. Participants' perceptions of the email and the customer support representative who wrote the email response were measured. In addition, participants were asked to evaluate how valued they felt by the response.

Various constructs such as the effectiveness of the response in resolving the consumer's problem, structure of the emails, quality of the emails, perception of CSR supportiveness, friendliness and respectfulness of the CSR, and perception of how valued the consumer felt, were measured by multiple items through factor analysis. Effectiveness of the email response was made up of the following items - the email summarized consumer's problem well, the email addressed consumer's concern, the email included relevant web links that consumer could click on for more information, the email provided the consumer with all the information needed, and the consumer found the email informative. The structure of email was composed of items including - the email was well structured, the format of the email made it easy to read, and extraneous information was not presented. The quality of the email was composed of items including - the email was well written, consistent, directed, straightforward, transparent, and had appropriate length.

The factor measuring supportiveness of CSR was composed of items including CSR responded to the consumer's concern adequately, understood the consumer's concern, provided the consumer with concrete steps to take, addressed the consumer's questions, was familiar with the consumer's situation, took steps to help resolve the consumer's situation, and was effective in responding to the consumer's question. The factor measuring perception of friendliness and respectfulness of the CSR included the items - the CSR was friendly, helpful, supportive, knowledgeable, and treated the consumer with respect. The factor measuring perceived empathy of CSR included the items - CSR was empathetic, firm, even-keeled, and no-nonsense.

The factor measuring how valued consumers felt by the email response was composed of the following items - the consumer felt valued, was treated fairly in this interaction, was addressed personally, felt good about being a consumer, felt that the company cared about the consumer's situation, and found the company's policies to be fair.

The factors were computed through principal components analysis. In the balanced questionnaire, the items that were framed with negative valence were reverse coded. The factors were highly reliable with Cronbach's alpha scores ranging from 0.77 to 0.93 .

\section{Results}

The data were analyzed employing multivariate analysis of variance. There were no significant interaction effects. As predicted, participants gave significantly higher 
responses on perceptions of the CSR, the email, and how valued they felt during the interaction in the affirmative statements condition compared to responses obtained through the balanced questionnaire.

\subsection{Questionnaire Type}

Participants rated the email as having solved the consumer's issue significantly more positively in the affirmative questionnaire compared to the balanced questionnaire $F(1,885)=198.15, p<0.0001, \eta^{2}=0.86, M=3.79 \& 3.13, S D=0.92 \& 0.41$ respectively. The effect size was high.

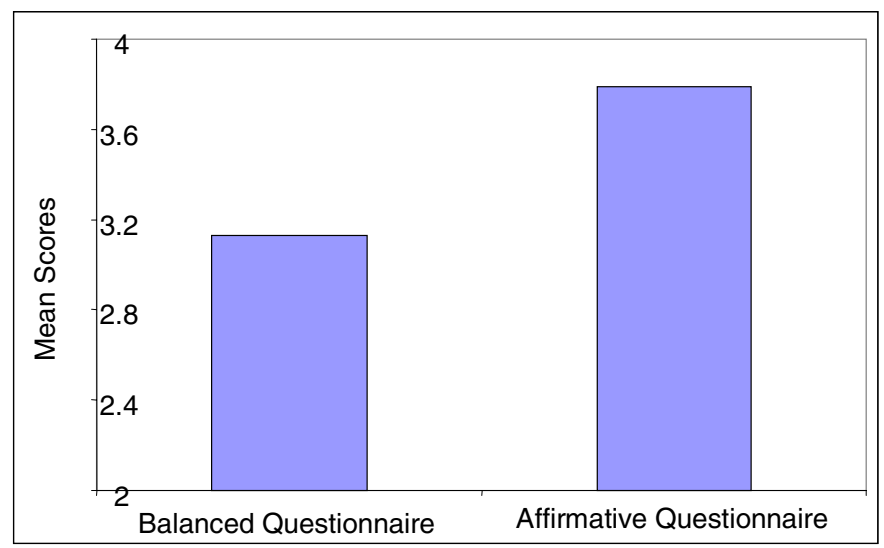

Fig. 1. Participants' rating of the effectiveness of the email response

Participants rated the structure of the email as being significantly better in the affirmative questionnaire compared to the balanced questionnaire $F(1,885)=304.86$, $p<0.0001, \eta^{2}=1.03, M=3.92 \& 3.12, S D=0.89 \& 0.39$ respectively. The effect size was extremely high.

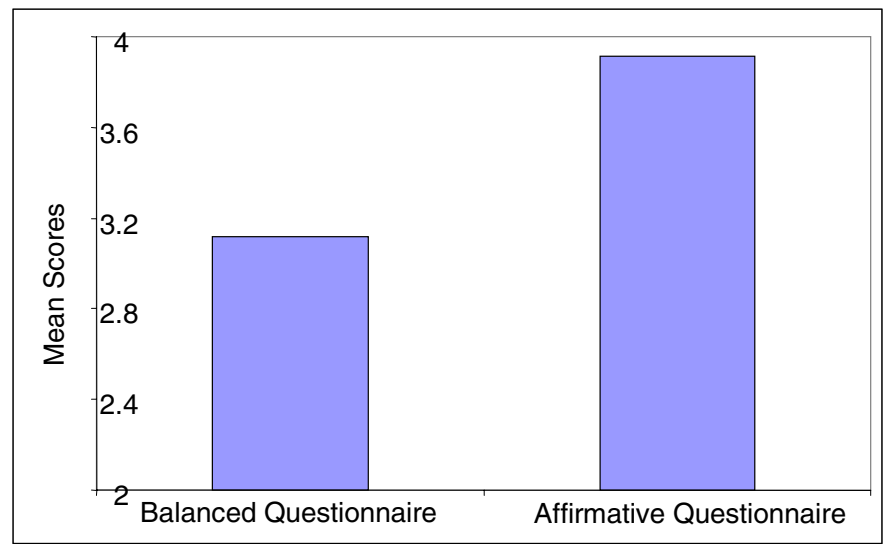

Fig. 2. Participants' evaluation of the structure of the email response 
Participants rated the quality of the email as being significantly better in the affirmative questionnaire compared to the balanced questionnaire $F(1,885)=31.99, p$ $<0.0001, \eta^{2}=0.38, M=3.80 \& 3.54, S D=0.81 \& 0.52$ respectively. The effect size was relatively low.

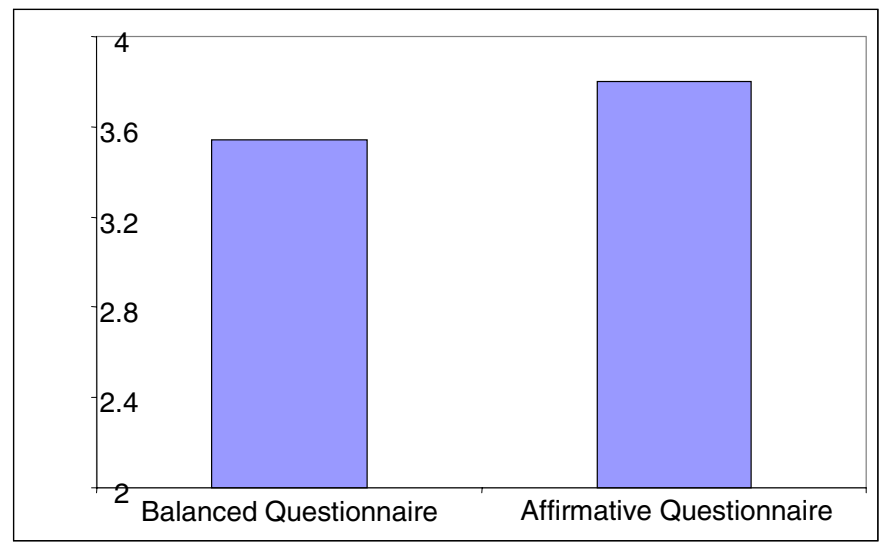

Fig. 3. Participants' evaluation of the quality of the email response

In particular, participants rated the email responder (in this case the CSR) as being significantly more supportive in the affirmative questionnaire than in the balanced questionnaire $F(1,885)=347.88, p<0.0001, \eta^{2}=1.06, M=3.77 \& 2.93, S D=0.93$ $\& 0.29$ respectively. The effect size was extremely high.

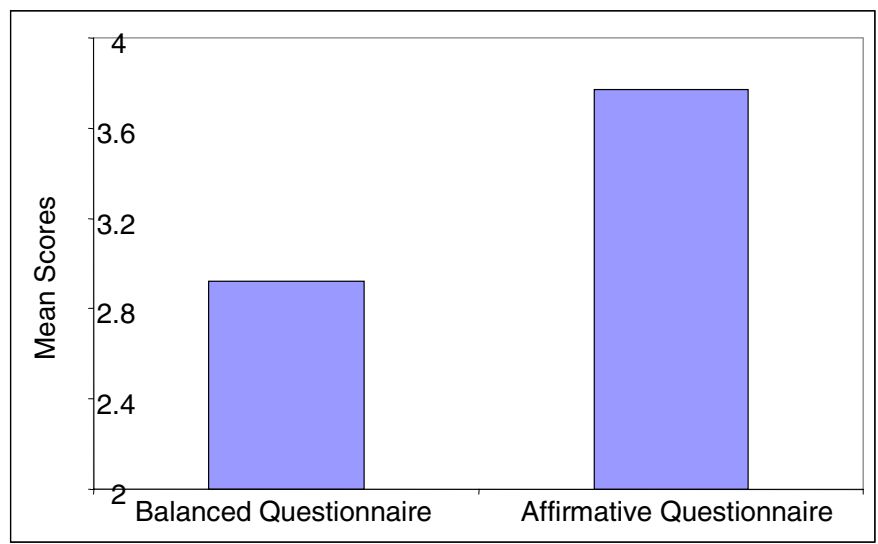

Fig. 4. Participants' evaluation of how supportive was the responder of the email

Participants rated the CSR responding to the consumer's question as being significantly friendlier and more respectful in the affirmative questionnaire compared 


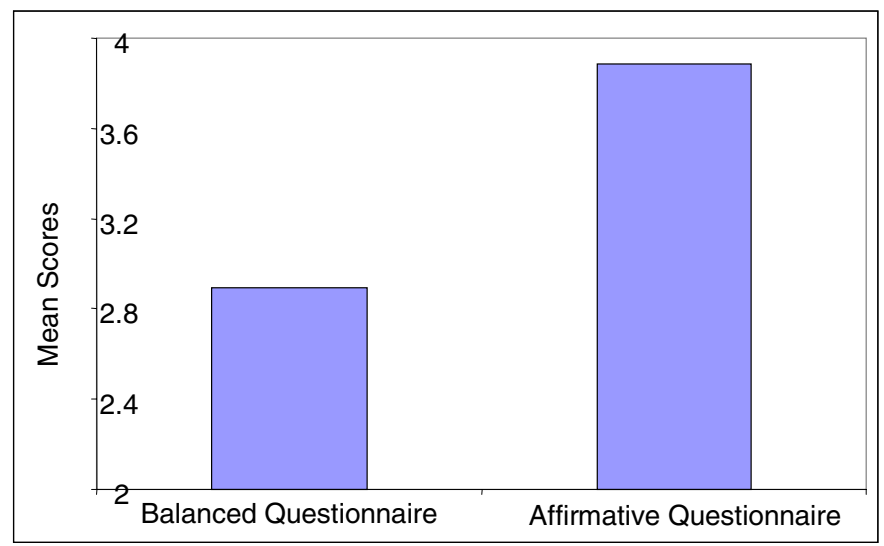

Fig. 5. Participants' evaluation of how respectful and friendly was the responder of the email

to the balanced questionnaire $F(1,885)=511.76, p<0.0001, \eta^{2}=1.23, M=3.89 \&$ $2.89, S D=0.88 \& 0.33$ respectively. The effect size was extremely high.

Participants rated the CSR as being significantly more empathetic in the affirmative questionnaire compared to the balanced questionnaire $F(1,885)=39.22, p$ $<0.0001, \eta^{2}=0.41, M=3.76 \& 3.45, S D=0.84 \& 0.62$ respectively. The effect size was moderate.

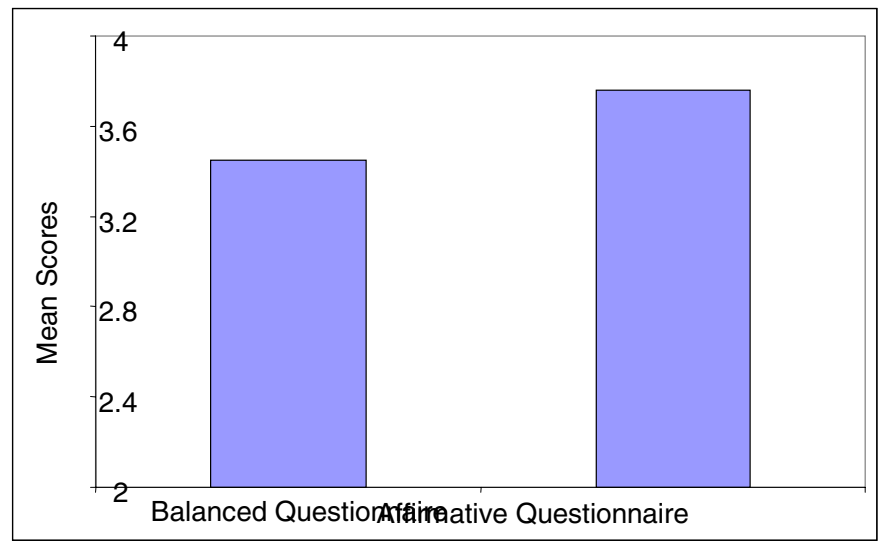

Fig. 6. Participants' evaluation of how empathetic was the responder of the email

Participants reported feeling significantly more valued by the CSR when they responded to the affirmative questionnaire compared to the balanced questionnaire $F(1,885)=72.03, p<0.0001, \eta^{2}=0.54, M=3.68 \& 3.25, S D=1 \& 0.40$ respectively. The effect size was moderate. 


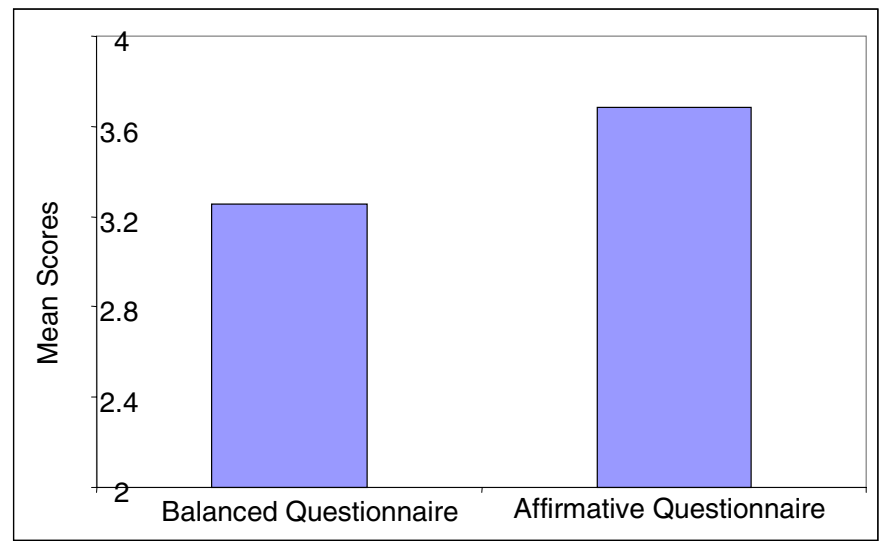

Fig. 7. Participants' evaluation of how valued they felt by the response

\subsection{Email Type}

Participants rated the email as having resolved the consumer's issue significantly more when the issue pertained to the company's billing policy compared to an issue with another user in the customer-to-customer e-commerce site $F(1,885)=14.44, p<$ $0.0001, \eta^{2}=0.23, M=3.53 \& 3.35, S D=0.77 \& 0.77$ respectively. The effect size was low.

Participants rated the structure of the email as being significantly better when the issue pertained to the company's billing policy compared to an issue with another user in the customer-to-customer e-commerce site $F(1,885)=4.6, p<0.0001, \eta^{2}=0.14$, $M=3.55 \& 3.44, S D=0.77 \& 0.79$ respectively. The effect size was low.

Participants rated the quality of the email as being significantly better when the issue pertained to the company's billing policy compared to an issue with another user in the customer-to-customer e-commerce site $F(1,885)=18.61, p<0.0001, \eta^{2}=$ $0.28, M=3.76 \& 3.57, S D=0.66 \& 0.69$ respectively. The effect size was low.

Participants rated the CSR as being significantly more supportive when the issue pertained to the company's billing policy compared to an issue with another member in the customer-to-customer e-commerce site $F(1,885)=8.05, p<0.0001, \eta^{2}=0.16$, $M=3.39 \& 3.26, S D=0.83 \& 0.76$ respectively. The effect size was low.

There was no difference in participants' rating of the CSR's friendliness and respectfulness in responding to the consumer's question when the issue pertained to the company's billing policy or when the issue pertained to a transaction problem with another user in the customer-to-customer e-commerce site $F(1,885)=2.85, p=$ $0.09, M=3.41 \& 3.32, S D=0.88 \& 0.76$ respectively.

There was no difference in participants' rating of the CSR's empathy in responding to the consumer's question when the issue pertained to the company's billing policy or when the issue pertained to a transaction problem with another user in the customer-to-customer e-commerce site $F(1,885)=3.67, p=0.06, M=3.65 \& 3.54$, $S D=0.75 \& 0.74$ respectively. 
Participants reported feeling significantly more valued by the CSR when they received a response to the company's billing policy related issue compared to the response received for an issue with another user in the customer-to-customer e-commerce site $F(1,885)=12.94, p<0.0001, \eta^{2}=0.33, M=3.55 \& 3.36, S D=$ $0.74 \& 0.80$ respectively. The effect size was low.

\section{Discussion}

When participants confirmed research hypotheses for the type of issue in the email, the effect sizes were low. Possibly, the statistical significance was the result of high power as a consequence of high cell-sizes.

Participants confirmed research hypotheses for the type of questionnaire employed to measure attitudes. In the case of framing statements in questionnaire design, when all the statements are framed positively, participants may assume that a positive response is sought by the researchers. Thus, they may try to be helpful by confirming what they believe to be the research hypotheses. Thus, respondents are likely to provide more positive responses in affirmative questionnaires. Clearly, responses to the affirmative questionnaire with only positive valence statements are affected by acquiescence bias.

In general, the effect sizes for the difference in attitudes between the balanced questionnaire and affirmative questionnaire were high both for the responder of the email and the artifact - email. The effect size for perception of self was somewhat lower. The smaller standard deviation for the balanced questionnaire compared to the questionnaire with items framed in only positive valence suggests that the respondents likely paid more attention to the individual items in the balanced questionnaire. Thus, the responses were probably more accurate reflections of their perceptions and attitudes, resulting in significantly less variability. Possibly, participants did not read the items as carefully in the questionnaire consisting of only positive valence items, thereby resulting in response set bias and greater variability. The reliability of the responses in both the questionnaires - balanced and affirmative, may be high. However, the validity of the outcome based on balanced questionnaire may be higher. Hence, assessments about the larger population based on the results obtained through balanced questionnaires would likely be more accurate. Consequently, user findings obtained with a balanced questionnaire administered to a random sample would have greater external validity, compared to findings derived from data collected though a questionnaire with items framed in only one direction.

\section{Conclusion}

Accurate measurement of attitudes is of paramount importance particularly in market and consumer research as inflated assessments of users' satisfaction or propensity to use products could have deleterious consequences in terms of investment in resources. Controlling for biases in the measurement of attitudes would provide stakeholders with a more accurate assessment, allowing them to take appropriate measures to improve user satisfaction, trust, and loyalty. 


\section{References}

1. Allport, G.W.: Attitudes. In: Murchison, C. (ed.) Handbook of Social Psychology, pp. 798884. Clark University Press, Worcester, MA (1935)

2. Brace, I.: Questionnaire Design: How to plan, structure and write survey material for effective market research. Kogan Page, London (2004)

3. Fishbein, M., Ajzen, I.: Belief, attitude, intention, and behavior: An introduction to theory and research. Addison-Wesley, Reading, MA (1975)

4. Krosnick, J.A.: Response strategies for coping with the cognitive demands of attitude measures in surveys. Applied Cognitive Psychology 5, 213-236 (1991)

5. Ray, J.J.: Reviving the problem of acquiescent response bias. The. Journal of Social Psychology 121, 81-96 (1983)

6. Reeves, B., Nass, C.: The Media Equation: How People Treat Computers, Television, and New Media Like Real People and Places. Cambridge University Press, Cambridge (1996)

7. Rorer, L.G.: The great response style myth. Psychological Bulletin 63, 129-156 (1965)

8. Tesser, A., Whitaker, D., Martin, L., Ward, D.: Attitude heritability, attitude change and physiological responsivity. Personality and Individual Differences 24, 89-96 (1998) 